\title{
Descripción Anatómica de la Musculatura Intrínseca y Cavidad de la Laringe en Cuatro Especies de Primates
}

\author{
Anatomical Description of the Intrinsic Musculature \\ and Cavity of the Larynx in Four Primate Species
}

Nicolle Villarroel-Villarroel \& Oliver Quintana-García

\begin{abstract}
VILLARROEL-VILLARROEL, N. \& QUINTANA-GARCÍA, O. Descripción anatómica de la musculatura intrínseca y cavidad de la laringe en cuatro especies de primates. Int. J. Morphol., 36(4):1326-1330, 2018.

RESUMEN: Los primates actualmente se clasifican en "Estrepsirrinos" y "Haplorrinos". Los estrepsirrinos habitan principalmente Madagascar y el sudeste de Asia, en cambio, los Haplorrinos se subdividen en "monos del viejo mundo" y "monos del nuevo mundo", distribuyéndose los primeros en África y Asia principalmente, mientras que los últimos tienen en Centro y Sudamérica sus principales áreas de distribución. Son animales frecuentes en zoológicos y centros de rehabilitación, que presentan abundantes estudios en cuanto a su distribución, hábitat, comportamiento y medidas de conservación, pero escasos son los estudios en relación a su anatomía. Por esta razón, el objetivo de nuestro estudio fue realizar una descripción anatómica de la musculatura intrínseca y cavidad de la laringe en cuatro ejemplares de primates, los cuales fueron donados por el Zoológico del Parque Metropolitano de Santiago de Chile, correspondientes a las especies: mono araña (Ateles fusciceps), mono capuchino (Cebus albifrons), gibón de manos blancas (Hylobates lar) y lémur cola anillada (Lémur catta). La disección se realizó desde superficial a profundo en cada laringe aislada, describiendo y comparando los hallazgos anatómicos observados, utilizando como guía comparativa la literatura anatómica humana. El estudio demostró diferencias anatómicas entre los primates en estudio, así como también diferencias con lo descrito en la literatura para la anatomía de la laringe humana.
\end{abstract}

PALABRAS CLAVE: Anatomía; Laringe; Primates.

\section{INTRODUCCIÓN}

Actualmente, los primates se clasifican en "Estrepsirrinos" y "Haplorrinos". Los Estrepsirrinos habitan principalmente Madagascar y el sudeste de Asia, en cambio, los Haplorrinos se subdividen en "Monos del viejo mundo" y "Monos del nuevo mundo", distribuyéndose los primeros en África y Asia principalmente, mientras que los últimos tienen en centro y Sudamérica sus principales áreas de distribución (Quevedo et al., 2009).

El mono araña (Ateles fusciceps) es una especie nativa en Colombia, Ecuador y Panamá. Están declarados en peligro crítico de extinción según la International Union for Conservation of Nature and Natural Resources (IUCN) debido al declive de la población, que es mayor al $80 \%$ en los últimos 45 años. A su vez, el mono capuchino (Cebus albifrons) es una especie nativa de Bolivia, Colombia, Perú y Venezuela. Se encuentra declarado como una especie de preocupación menor, por ser una especie abundante y de amplia distribución, pero su población actual ha ido decreciendo, siendo una de las razones por las que su comercio debe ser controlado para evitar un uso incompatible con su supervivencia. El gibón de manos blancas (Hylobates lar) es una especie nativa en Indonesia, República Democrática Popular Lao, Malasia, Tailandia y República de la Unión de Myanmar. Actualmente, está catalogado como una especie en peligro de extinción y está prohibido su comercio, el que se autoriza solamente bajo circunstancias excepcionales. Por último, el lémur cola anillada (Lémur catta) es una especie nativa de Madagascar, y se encuentra como todos los lémures en peligro de extinción. Su comercio se encuentra prohibido desde 1977. Fenómenos como la fragmentación y transformación del hábitat en zonas agrícolas y ganaderas, la cacería ilegal y el comercio han provocado que estas especies se encuentren en riesgo (Albuja \& Arcos, 2007; International Union for Conservation of Nature and Natural Resources, 2016; Convention on International Trade in Endangered Species of Wild Fauna and Flora, 2016).

Los primates son animales frecuentes en zoológicos porque muestran conductas y estructuras sociales similares a las de los humanos (López \& Tárano, 2006). Por lo tanto, se necesitan manejos diarios donde es fundamental 
conocer diferentes aspectos de ellos, tales como su anatomía. Estas son especies bastantes estudiadas en el ámbito de la distribución, hábitat, comportamiento y medidas de conservación, pero lamentablemente hay pocos estudios relacionados con la anatomía descriptiva de estos animales, por lo que, es una gran contribución realizar estudios sobre su morfología. Los primates comparten más del 90 $\%$ del ADN con los humanos, siendo taxonómicamente similares (Mudry \& Nieves, 2010).

La función del sistema respiratorio es asegurar el intercambio gaseoso entre el aire atmosférico y la sangre. Está compuesto por las vías respiratorias, las cuales son recorridas por el aire inspirado y espirado, y los pulmones, tejido encargado de realizar el intercambio gaseoso. Las vías respiratorias comprenden: la nariz y cavidades nasales, la laringe, tráquea y los bronquios (Guyton \& Hall, 1996).

La laringe corresponde a un corto conducto formado por un conjunto de cartílagos, ligamentos y músculos, con importantes funciones, como permitir el paso del aire desde o hacia la tráquea cervical y evitar el paso de cuerpos extraños hacia las vías aéreas caudales (Evans \& deLaHunta, 2002; Concha, 2010).

\section{MATERIAL Y MÉTODO}

Se evaluaron cuatro laringes pertenecientes a ejemplares de primates de las especies: mono araña (Ateles fusciceps), mono capuchino (Cebus albifrons), gibón de manos blancas (Hylobates lar) y lémur de cola anillada (Lemur catta), los cuales fueron donados a la Unidad de Anatomía Veterinaria de la Universidad Santo Tomás, sede Santiago, campus Catemito por el Zoológico del Parque Metropolitano de Santiago, Chile.

Los ejemplares fueron conservados en congelación hasta la fecha del estudio. Posterior a su descongelación a temperatura ambiente, cada primate se posicionó en decúbito dorsal para remover la laringe a través de una incisión en la línea mediana ventral del cuello para luego separarla de las estructuras adyacentes. Previo a la disección, las laringes aisladas se conservaron inmersas en solución de formalina al $3 \%$ para su estudio y descripción.

Los músculos intrínsecos se disecaron desde superficial a profundo. Posteriormente, se realizó una incisión sagital dorsal para observar la cavidad laríngea en sus tres niveles: una superior, supraglótica, denominada vestíbulo laríngeo; una zona media, glotis; una zona inferior, subglótica, la cavidad infraglótica.

\section{RESULTADOS Y DISCUSIÓN}

Las laringes del mono araña y gibón de manos blancas midieron $3 \mathrm{~cm}$ de largo desde el vértice de la epiglotis hasta la unión del cartílago cricoides con el primer anillo traqueal, mientras que las laringes del lémur cola anillada y mono capuchino midieron $2 \mathrm{~cm}$ de largo. No fue posible realizar una relación relativa al peso corporal total de cada primate, dado que los especímenes fueron donados posterior al proceso de necropsia realizado por personal del Zoológico del Parque Metropolitano de Santiago, donde se removieron órganos de las cavidades torácica y abdominal.

\section{Músculos intrínsecos de la laringe}

\section{Músculos superficiales ventrales}

Músculo cricotiroideo: Presente en las cuatro especies, músculo par, ubicado caudo-ventralmente en la laringe, profundo a un músculo extrínseco, el músculo esternohioídeo. En el mono capuchino, gibón de manos blancas y lémur cola anillada se originan en la cara ventro-lateral del margen caudal del cartílago cricoides para seguir su recorrido hasta llegar a la superficie caudo-medial del cartílago tiroides, destacando su forma triangular. En cambio, en el mono araña presenta el mismo origen, pero se inserta en el margen craneal de la lámina del cartílago tiroides, presentando una forma rectangular. Según Latarjet \& Ruiz Liard (2010), en el humano, se describe una porción recta y oblicua de este músculo, las cuales no se lograron diferenciar en ninguna de las cuatro laringes en estudio. Además, se observó la separación de los músculos derecho e izquierdo, formando una "V" laríngea en el mono capuchino y mono araña, a diferencia de la laringe del lémur cola anillada y el gibón de manos blancas, en los cuales no se observó este espacio, ya que las fibras derechas e izquierdas se encuentran bastantes próximas entre sí (Fig. 1).

\section{Músculos superficiales dorsales}

Músculo cricoaritenoideo dorsal: Presente en las cuatro especies, es un músculo par con una forma triangular. Se origina en la superficie dorsal de la lámina del cartílago cricoides para insertarse en el proceso muscular del cartílago aritenoides. En el mono araña, gibón de manos blancas y mono capuchino se observó un espacio entre músculos derecho e izquierdo, a diferencia del lémur cola anillada en el cual no se observó este espacio (Fig. 2).

Músculo aritenoideo oblicuo: Descrito en el humano (Latarjet \& Ruiz Liard). Sin embargo, no se logró identificar en de los primates, posiblemente porque sus fibras se encuentran unidas al músculo aritenoideo transverso, o simplemente porque está ausente. 


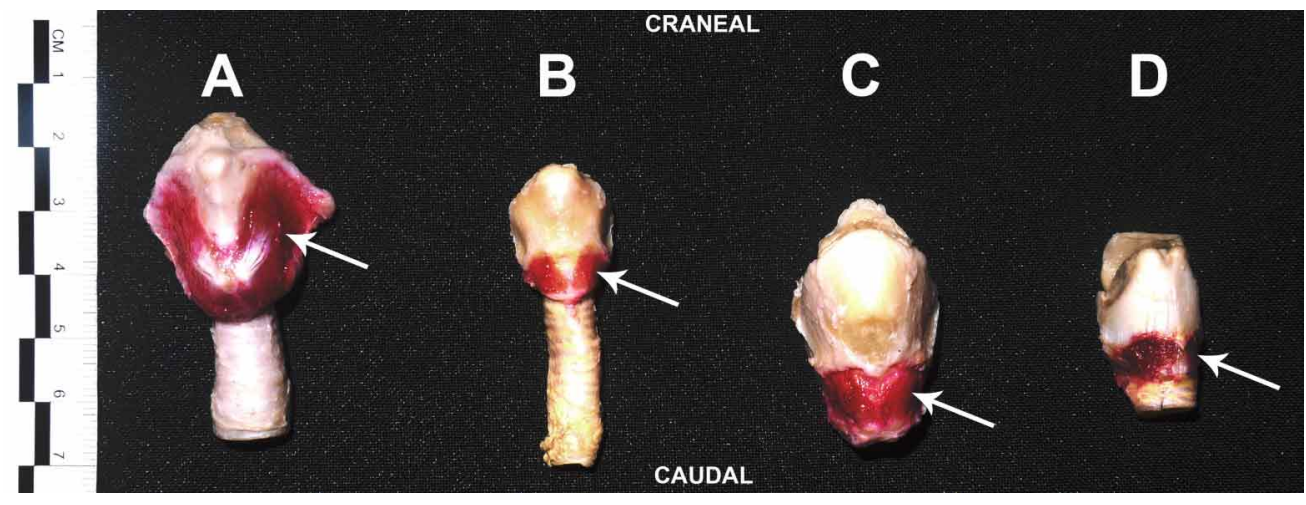

Fig. 1. Vista ventral de la laringe, con regla métrica al lado izquierdo, músculos cricotiroideos indicados con las flechas blancas: A. Mono araña, B. Gibón de manos blancas, C. Lémur cola anillada y D. Mono capuchino.

Músculo aritenoideo transverso: Está presente en el lémur cola anillada, gibón de manos blancas y mono araña. No se observó en el mono capuchino (Fig. 2). Es un músculo impar, concordando con lo propuesto por Latarjet \& Ruiz Liard para el humano, al igual que lo mencionado por Sisson \& Grossman (1982), en la anatomía veterinaria de especies domésticas. Se origina desde el engrosamiento existente entre la superficie lateral y dorsal del cartílago aritenoides y transversalmente cruza la porción dorsal de la laringe para insertarse dentro del engrosamiento que hay entre las superficies dorsal y lateral del cartílago aritenoides opuesto, coincidiendo con lo descrito por Rouvière \& Delmas (2005).

\section{Músculos profundos laterales}

Músculo tiroaritenoideo: Músculo par que se observó en las cuatro especies estudiadas. Se origina a lo largo de la

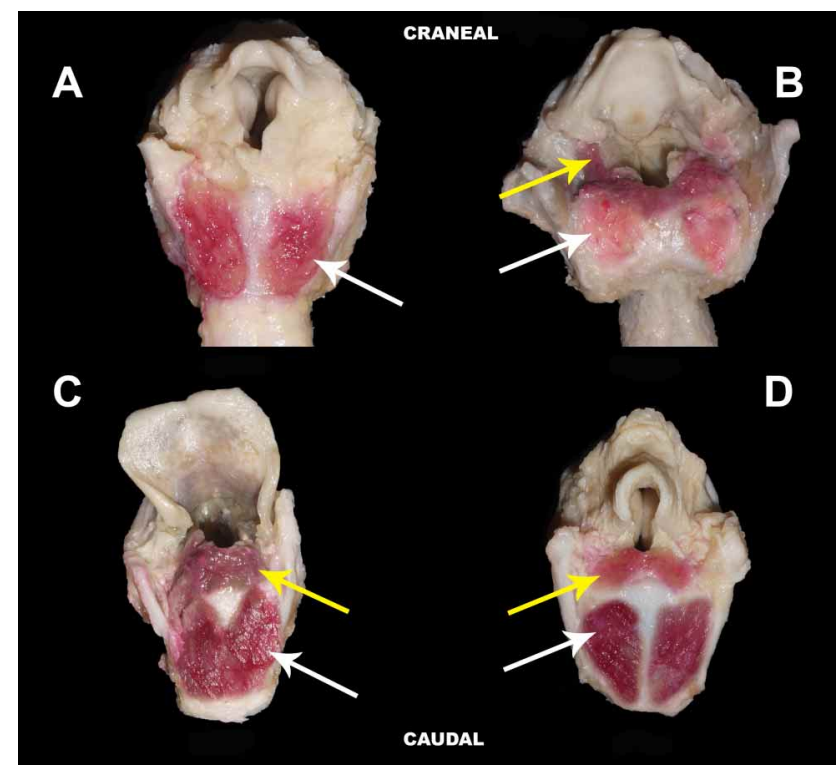

Fig. 2. Vista dorsal de la laringe, músculos cricoaritenoideos dorsales indicados con flecha blanca y músculo aritenoideo transverso indicado con flecha amarilla: A. Mono capuchino, B. Mono araña, C. Lémur cola anillada y D. Gibón de manos blancas. línea mediana interna del cartílago tiroides, sus fibras cursan caudo-dorsalmente para insertarse en el cartílago aritenoides en el rafe del origen del músculo aritenoides transverso. De acuerdo a Latarjet \& Ruiz Liard, se le describen a este músculo tres divisiones: un fascículo superior, fascículo medio y fascículo inferior, los cuales no se lograron identificar en ninguno de los cuatro primates estudiados, posiblemente por el tiempo prolongado de conservación de los ejemplares en estudio (congelación), lo que podría haber provocado un deterioro de las fibras o simplemente porque no presentaban dicha división. También se describe la porción tiroepiglótica, la cual tiene fibras orientadas hacia el cartílago epiglótico, que sólo se logró reconocer en lémur cola anillada y gibón de manos blancas (Fig. 3).

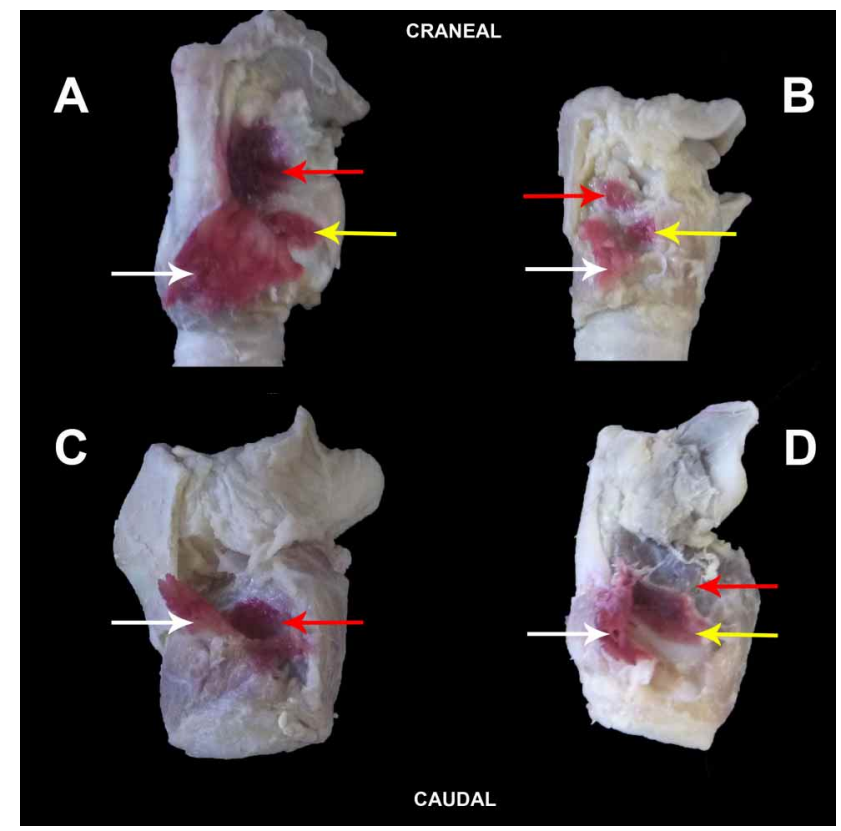

Fig. 3. Vista lateral izquierda de la laringe, músculo tiroaritenoideo indicado con flecha blanca, músculo cricotiroideo lateral indicado con flecha amarilla y músculo vocal indicado con la flecha roja: A. Mono araña, B. Mono capuchino, C. Gibón de manos blancas y D. Lémur cola anillada. 
Músculo cricotiroideo lateral: Es par, con forma cuadrilátera irregular. Se identificaron fácilmente sus fibras en el lémur cola anillada, mono capuchino y mono araña, ya que, al momento de disecar se separaron sin dificultad del músculo tiroaritenoideo adyacente. Se origina en el margen craneal de la porción lateral del arco del cartílago cricoides y continúa dorsalmente para insertarse en el cartílago aritenoides a un lado del proceso muscular. En cambio, en el gibón de manos blancas no se logró identificar, se puede plantear que el músculo se encontraba adosado al músculo tiroaritenoideo adyacente, o que simplemente esta especie no posee este músculo (Fig. 3)

Músculo vocal: Observado en las cuatro especies, presenta una función muy importante debido a que relaja los pliegues vestibulares (Latarjet \& Ruiz Liard). Presentó diferentes formas en las distintas especies, posiblemente porque este músculo se encuentra inmediatamente profundo al músculo tiroaritenoideo y pueden estar adosadas fibras de este músculo en el músculo vocal. Se origina en la línea mediana interna del cartílago tiroides y se inserta en el proceso vocal del cartílago aritenoides (Fig. 3).

Cavidad de la laringe: La cavidad laríngea posee una forma similar a un reloj de arena, la porción más estrecha en su parte media está formada por los pliegues vestibulares y vocales. Esta estrechez permite dividir la cavidad en tres zonas: una superior, supraglótica, denominada vestíbulo laríngeo; una zona media, glotis; una zona inferior, subglótica, la cavidad infraglótica.

Zona supraglótica, Vestíbulo laríngeo: Se extiende desde el aditus laríngeo hasta el pliegue vestibular, que es un pliegue par de la mucosa situado en las paredes laterales de la cavidad. Se observaron diferencias en el vértice epiglótico entre las especies tales como: el lémur presentó una forma cuadrada, de gran tamaño y ancha. En el gibón y mono capuchino se observó un vértice epiglótico con forma redondeada, pequeño y con una incisura poco profunda en la porción más craneal. El mono araña presentó un vértice de forma redondeada, pero de gran tamaño (Fig. 4).

Zona glótica, Glotis: Consta de pliegues vestibulares, pliegues vocales y la hendidura glótica, que es el estrecho canal que atraviesa la glotis. Los pliegues vestibulares (cuerdas vocales superiores o falsas) se observaron en las cuatro especies de primates: el gibón, mono araña y mono capuchino presentaron los pliegues vestibulares más desarrollados y engrosados, a diferencia del lémur donde se observaron de menor tamaño y delgados, de la misma proporción que los pliegues vocales. Los ventrículos laríngeos son divertículos de la cavidad laríngea, desarrollados hacia las paredes laterales entre los pliegues vestibulares y vocales. No se observaron grandes diferencias entre las especies en estudio, solo en el lémur cola anillada, en el que se observó la entrada al ventrículo laríngeo de características más amplias. Los pliegues vocales (cuerdas vocales inferiores o verdaderas) se observaron en las cuatro especies con dimensiones muy similares y conservadas, ubicados entre el proceso vocal del cartílago aritenoides y la parte mediana ventral del cartílago tiroides (Fig. 4).

Zona subglótica, Cavidad infraglótica: Ventral a la hendidura glótica, la cavidad laríngea se ensancha hasta las dimensiones de la circunferencia interna del anillo cricoides. Comunica hacia caudal con la tráquea cervical. No se observaron diferencias a destacar en esta zona.

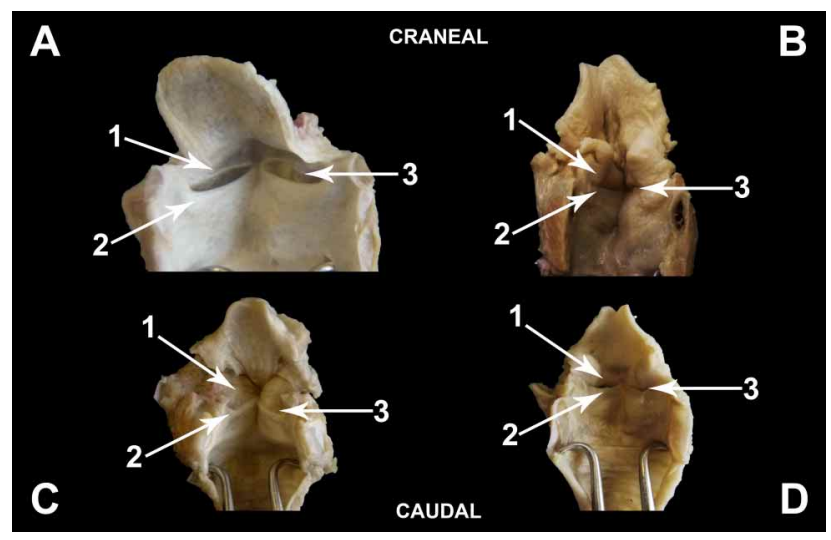

Fig. 4. Cavidad laríngea con sección sagital en su pared dorsal, vista dorsal. Desplazamiento lateral de las paredes con ayuda de instrumental quirúrgico para observar estructuras de la cavidad de la laringe: A Lémur cola anillada, B Gibón de manos blancas, C Mono araña y D Mono capuchino. 1 Pliegue vestibular, 2 Pliegue vocal y 3 Ventrículo laríngeo.

\section{CONCLUSIONES}

La laringe corresponde a un importante órgano tubular que compone parte de las vías respiratorias. Situado entre la faringe dorsalmente y la tráquea inferiormente, es el encargado de evitar el paso de cuerpos extraños y proteger la entrada a las vías respiratorias inferiores. Además, es el encargado de la fonación, proceso por el cual se modifica la corriente de aire que se origina desde los pulmones para emitir diferentes tipos de sonidos o vocalizaciones, las cuales representan un medio de comunicación importante entre los animales.

De los músculos intrínsecos de la laringe, el cricotiroideo del mono capuchino y mono araña son similares a lo observado en el hombre, originando la "V" laríngea y con forma triangular en las 4 especies. El músculo cricoaritenoideo dorsal se observó en todas las especies, destacando el espacio entre el derecho e izquierdo en el mono araña, capuchino y gibón de manos blancas. No se identificó 
el músculo aritenoideo oblicuo en ningún primate, el cual si está descrito para el humano. El músculo aritenoideo transverso presenta la porción tiroepiglótica en el mono araña y lémur cola anillada, similar a lo descrito para el humano y contrario a lo observado en el mono capuchino, en el cual se encuentra ausente. El músculo tiroaritenoideo se observó en las cuatro especies de primates similar a lo descrito para el humano al igual que el musculo vocal. El músculo cricoaritenoideo lateral se identificó fácilmente en el mono araña y lémur, pero se encontró ausente en el gibón de manos blancas.

La configuración cavitaria de la laringe presentó diferencias principalmente en la forma del vértice epiglótico, el cual se observó redondeado en el mono araña, capuchino y gibón de manos blancas. El pliegue vestibular se observó de mayor tamaño en el lémur cola anillada, al igual que la entrada a los ventrículos laríngeos.

A pesar de ser primates, que están emparentados taxonómicamente, cada uno presenta distintos comportamientos, hábitats, alimentación y hábitos de vida que podrían llegar a explicar las diferencias anatómicas observadas entre las cuatro especies, así como también para lo descrito con el humano, el cual sabemos, ha logrado un gran desarrollo en la emisión de sonidos inteligibles que nos permiten la comunicación oral.

Los resultados de este estudio sugieren una mayor cantidad de diferencias con relación a la musculatura intrínseca en comparación a la configuración cavitaria de la laringe entre las cuatro especies, lo cual debe ser considerado al momento de realizar algún abordaje de tipo quirúrgico, imagenológico o simplemente la instalación de una vía aérea en el órgano estudiado.

VILLARROEL-VILLARROEL, N. \& QUINTANA-GARCÍA, O. Anatomical description of the intrinsic musculature and cavity of the larynx in four primate species. Int. J. Morphol., 36(4):13261330, 2018.

SUMMARY: Primates are currently classified as "Strepsirrhini" and "Haplorrhini". The strepsirrhines inhabit mainly Madagascar and Southeast Asia. However, the Haplorrhini are subdivided into "old world monkeys" and "new world monkeys", the first being distributed in Africa and Asia mainly, while the main distribution areas for the latter are in Central and South America. They are frequent animals in zoos and rehabilitation centers, allowing abundant studies regarding their distribution, habitat, behavior and conservation measures, but few studies are related to their anatomy. For this reason, the aim of our study was to perform an anatomical description of the intrinsic muscles and cavity of the larynx in four specimens of primates, which were donated by the Metropolitan Park of Santiago, Chile, corresponding to species: spider monkey (Ateles fusciceps), capuchin monkey (Cebus albifrons), white-handed gibbon (Hylobates lar) and ring-tailed lemur (Lemur catta). The dissection was performed from superficial to deep in each isolated larynx, describing and comparing the observed anatomical findings, using as a comparative guide the human anatomical literature. The study showed anatomical differences between the primates under study, as well as differences for what is described in the literature for the anatomy of the human larynx.

KEY WORDS: Anatomía; Laringe; Primates.

\section{REFERENCIAS BIBLIOGRÁFICAS}

Albuja, L. \& Arcos, R. Evaluación de las poblaciones de Cebus albifrons cf. Aequatorialis en los bosques suroccidentales ecuatorianos. Politécnica, 27(4):58-67, 2007.

Concha, I. Anatomía del Perro. Santiago de Chile, Universidad Santo Tomás, 2012.

Convention on International Trade in Endangered Species of Wild Fauna and Flora (CITES). Ginebra, Convention on International Trade in Endangered Species of Wild Fauna and Flora, 2016. Disponible en: https://cites.org/esp/app/appendices.php

Evans, H. \& deLaHunta, A. Disección del Perro. $5^{\mathrm{a}}$ ed. New York, McGrawHill Interamericana, 2002.

Guyton, A. C \& Hall, J. E. Tratado de Fisiología Médica. $9^{\mathrm{a}}$ ed. Madrid, Interamericana McGraw-Hill, 1996.

International Union for Conservation of Nature and Natural Resources (IUCN). The IUCN Red List of Threatened Species. Cambridge, International Union for Conservation of Nature and Natural Resources, 2016. Disponible en: http://www.iucnredlist.org

Latarjet, M. \& Ruiz Liard, A. Anatomía Humana. 4a ed. Buenos Aires, Médica Panamericana, 2010.

López, M. C. \& Tárano, Z. Comportamiento social del mono capuchino común Cebus olivaceus (Primates: Cebidae) en tres exhibiciones zoológicas de Caracas, Venezuela. Rev. Biol. Trop., 56(3):1503-20, 2006.

Mudry, M. D. \& Nieves, M. Primates sin fronteras: una visión desde la citogenética evolutiva. B. A. G. J. Basic Appl. Genet., 21(2), 2010. Disponible en: http://ppct.caicyt.gov.ar/index.php/bag/article/view/49/ 161

Quevedo, U. M.; Cisneros, S. J.; Navarette, Z. M.; Torres, G. J. P. \& Sato, S. A. Descripción anatómica de los músculos del miembro posterior y cola del mono machín blanco (Cebus albifrons). Rev. Investig. Vet. Perú, 20(2):165-70, 2009

Rouvière, H. \& Delmas, A. Anatomía Humana: Descriptiva, Topográfica y Funcional. 11 ${ }^{\mathrm{a}}$ Ed. Barcelona, Elsevier Masson, 2005.

Sisson, S. \& Grossman, J. Anatomía de los Animales Domésticos. 5a ed. Bogotá, Elsevier Masson, 1982.

Dirección para correspondencia

Dr. Oliver Quintana García

Universidad Santo Tomás

Camino Catemito 1830, San Bernardo

Santiago - CHILE

Email: oquintanag@santotomas.cl 